\title{
Il Bioventing: una tecnologia che usa i microrganismi per degradare gli inquinanti nei terreni contaminati da idrocarburi
}

\author{
Maddalena Gargione ${ }^{1}$ \\ ${ }^{1}$ University of Salerno
}

\begin{abstract}
La bonifica dei siti contaminati è una delle problematiche più rilevanti nell'ambito degli interventi di recupero e di risanamento ambientale. Si definiscono "contaminate" quelle aree in cui, a causa di sversamenti accidentali, depositi abusivi ma anche condizioni naturali, sono presenti nel suolo sostanze inquinanti tali da risultare pericolose per l'uomo e per l'ambiente. Negli ultimi decenni si sono sviluppate alcune tecniche di bonifica che sfruttano la degradazione dei contaminanti ad opera di microrganismi con lo scopo di arrecare un minore impatto sull'ecosistema e un considerevole risparmio economico. Tra le molteplici tecniche trova spazio il Bioventing, una tecnologia adoperata nella zona insatura del terreno che consente l'utilizzo di microrganismi autoctoni per la degradazione di composti organici adsorbiti dalle frazioni minerali e organiche del suolo. In questo articolo si fa riferimento ad un caso studio che ha trattato la tecnica del Bioventing per la rimozione di idrocarburi del petrolio.
\end{abstract}

\section{Bioventing: principi fondamentali}

Il Titolo V del Decreto Legislativo 3 aprile 2006, n. 152 "Norme in materia ambientale", successivamente modificato ed integrato dal Decreto Legislativo 16 gennaio 2008 n. 4, costituisce il riferimento legislativo nazionale per gli interventi di bonifica e ripristino ambientale dei siti conta- 
minati. Il Bioventing è un intervento di bonifica in situ che utilizza i microrganismi naturalmente presenti nel suolo per biodegradare i composti organici adsorbiti nella zona parzialmente satura del terreno. In alcuni casi la biodegradazione può avvenire naturalmente, in altri casi è necessario andare a stimolare l'attività microbica per favorire la degradazione delle sostanze. I microrganismi sono il fattore chiave nel degrado delle molecole naturali, però bisogna tener conto degli impatti che tali ceppi possono subire a causa dell'inquinamento ${ }^{1}$. L'attività dei microrganismi con il Bioventing, infatti, viene rafforzata introducendo aria atmosferica attraverso dei pozzi di iniezione o estrazione e, se necessario, aggiungendo sostanze nutritive. In genere tale tecnica viene adoperata per la degradazione di sostanze di medio peso come gli idrocarburi del petrolio (PHC). Essi, infatti, sono da sempre naturalmente presenti nell'ambiente, di conseguenza molti microrganismi hanno una naturale capacità di degradarli ${ }^{1}$. Infatti, in molti siti dove è presente una contaminazione da idrocarburi, esistono batteri autoctoni presenti nel terreno che possono metabolizzare l'idrocarburo, la cui attività è però limitata dalla mancanza di ossigeno. Il Bioventing entra in gioco appositamente per favorire l'immissione di ossigeno e garantire una degradazione in condizioni aerobiche degli inquinanti.

\section{Il Bioventing come tecnologia adoperata per la rimozione di}

\section{PHC}

Il Bioventing è una tecnica adoperata per bonificare i terreni contaminati da PHC, poiché migliora la capacità dei microrganismi di degradare tali sostanze. Il caso studio analizzato tratta la valutazione di tale tecnologia per risanare un terreno contaminato da benzina e benzina-etanolo ${ }^{1}$. Sono stati prelevati dei campioni di terreno indisturbati e in condizioni asettiche con lo scopo di analizzarne le caratteristiche geotecniche, chimiche, mineralogiche e microbiologiche. I blocchi di terreno ana- 
lizzati sono di dimensioni cubiche (con lato $30 \mathrm{~cm}$ ) e, al momento del prelievo, sono stati avvolti in fogli di alluminio, pellicola di plastica e paraffina, per evitare modifiche relative all'umidità del campione, infine sono stati lasciati in una stanza umida fino al momento del test. In totale sono stati prelevati 6 blocchi, di cui uno è stato analizzato senza trattamento, uno è stato sottoposto al Bioventing senza essere contaminato, due sono stati contaminati da benzina e benzina-etanolo senza essere trattati e gli ultimi due invece sono stati contaminati da benzina e benzina-etanolo per poi essere trattati con il Bioventing. Nei blocchi in cui è previsto il trattamento è stato iniettato un flusso d'aria di 2 psi, in particolare, per i blocchi contaminati, tale intervento è avvenuto dopo 10 giorni dalla contaminazione. Sono stati adoperati 10 litri di contaminante, suddiviso in benzina e benzina modificata con etanolo al $22 \%$, da versare sui vari blocchi tenendo conto di non modificare le condizioni iniziali di umidità. I microrganismi nel terreno nella prova in esame, sono stati analizzati nelle condizioni iniziali, dopo 24 ore, 30 giorni e 100 giorni dalla contaminazione. I risultati hanno evidenziato comportamenti differenti nei blocchi contaminati da sola benzina e quelli contaminati da benzina-etanolo. In particolare, per il terreno ventilato con benzina-etanolo si è registrata una degradazione importante ( $>98 \%)$, mentre quelli non ventilati hanno presentato una degradazione inferiore (74\%). Nei terreni invece contaminati da sola benzina il suolo ventilato nei primi 60 giorni ha raggiunto una degradazione leggermente inferiore di quello non ventilato. Ciò ha dimostrato che il Bioventing ha un effetto benefico nei terreni in cui è presente l'etanolo poiché ha accelerato la bonifica del suolo. Tale studio tuttavia ha mostrato un effetto minore nel suolo contaminato da benzina. Questo caso studio è stato preso in considerazione poiché gli stessi esperti ritengono che i dati ottenuti in laboratorio sono paragonabili a quelli delle misurazioni sul campo, di conseguenza tali analisi possono essere un buon riferimento nella pratica ${ }^{1}$. La tecnologia del Bioventing è tutt'ora analizzata per la rimozione di ulteriori composti da idrocarburi ${ }^{2}$ come toluene ${ }^{3}$, fenantrene ${ }^{4}$, carburante da serbatoi sotterranei ${ }^{5}, \mathrm{PCE}^{6}$, DDT e DNT ${ }^{7}$. 


\section{Conclusioni}

Il caso studio analizzato ha permesso di comprendere come il Bioventing sia una tecnologia adatta alla rimozione di idrocarburi $(\mathrm{PHC})$ nei terreni contaminati. In particolare, si è evidenziata una capacità di degradazione abbastanza elevata per i terreni contaminati da benzina-etanolo, dati più modesti invece si riferiscono ai terreni contaminati da sola benzina. In sostanza la tecnica del Bioventing ha portato per tutti i campioni degli ottimi risultati in termini di rimozione dei contaminanti, anche se in tempi più lunghi. C'è da evidenziare che i tassi di biodegradazione del PHC ottenuti dagli esperti in condizioni di laboratorio possono essere di riferimento per i casi pratici. La tecnologia del Bioventing può essere considerata quindi come un riferimento per la rimozione degli idrocarburi del petrolio, anche perchè risulta relativamente economica, soprattutto quando associata al SVE, in questo caso infatti è possibile utilizzare la medesima soluzione impiantistica per l'aspirazione e l'immissione di aria atmosferica.

\section{References}

1.Osterreichercunha, P. Evaluation of bioventing on a gasoline-ethanol contaminated undisturbed residual soil. Journal of Hazardous Materials 110, 63-76 (2004).

2.Hoeppel, R. E., Hinchee, R. E. \& Arthur, M. F. Bioventing soils contaminated with petroleum hydrocarbons. Journal of Industrial Microbiology 8, 141-146 (1991).

3.SUI, H. \& LI, X. Modeling for Volatilization and Bioremediation of Toluene-contaminated Soil by Bioventing. Chinese Journal of Chemical Engineering 19, 340-348 (2011).

4.Frutos, F. J. G., Escolano, O., García, S., Babín, M. \& Fernández, M. D. Bioventing remediation and ecotoxicity evaluation of phenanthrene-contaminated soil. Journal of Hazardous Materials 183, 806-813 (2010). 
5.Kampbell, D. H. \& Wilson, J. T. Bioventing to treat fuel spills from underground storage tanks. Journal of Hazardous Materials 28, 75-80 (1991).

6.Mihopoulos, P. Vapor phase treatment of PCE in a soil column by lab-scale anaerobic bioventing. Water Research 34, 3231-3237 (2000).

7.Shah, J. K., Sayles, G. D., Suidan, M. T., Mihopoulos, P. \& Kaskassian, S. Anaerobic bioventing of unsaturated zone contaminated with DDT and DNT. Water Science and Technology 43, 35-42 (2001). 
Figure Captions

Figure 1. Contaminazione da idrocarburi del petrolio 
Figures

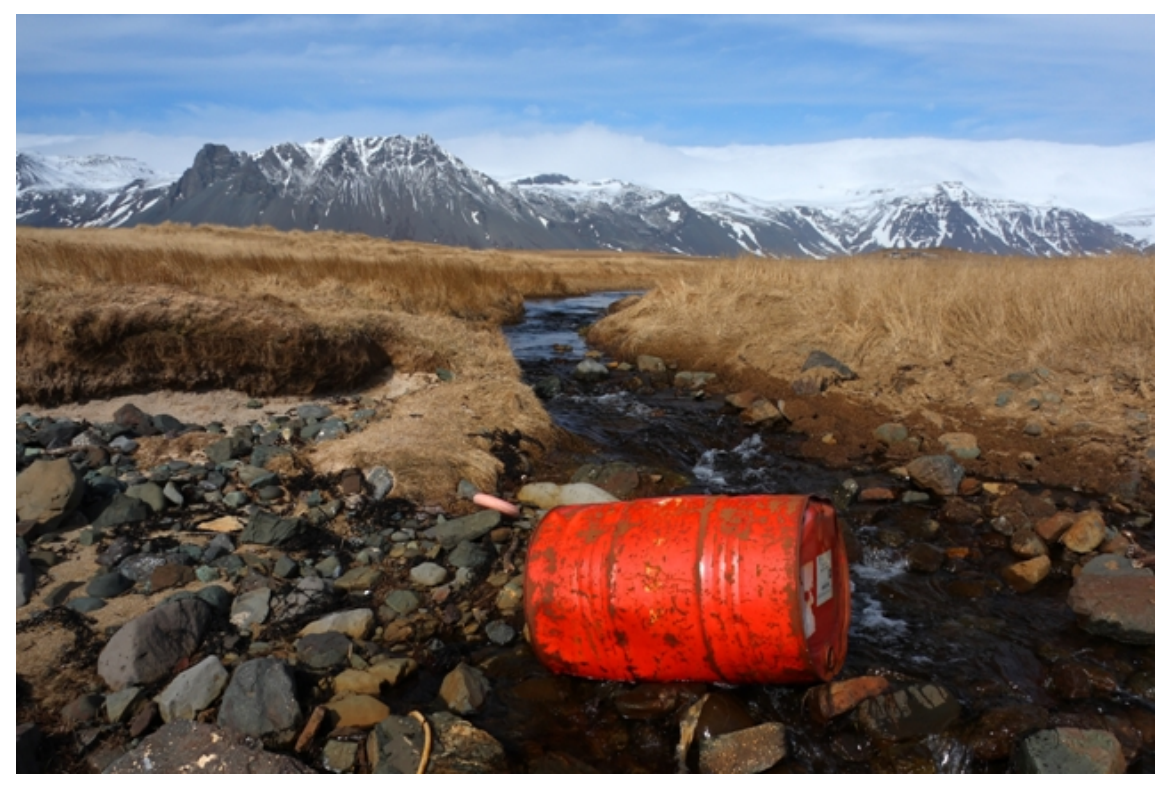

Figure 1: Contaminazione da idrocarburi del petrolio 